\title{
Hurricane Season Public Health Preparedness, Response, and Recovery Guidance for Health Care Providers, Response and Recovery Workers, and Affected Communities - CDC, 2017
}

\author{
CDC 2017 Hurricane Incident Management System Team ${ }^{1}$
}

\section{On September 13, 2017, this report was posted as an MMWR Early Release on the MMWR website (https://www.cdc.gov/mmwr).}

CDC and the Agency for Toxic Substances and Disease Registry (ATSDR) have guidance and technical materials available in both English and Spanish to help communities prepare for hurricanes and floods (Table 1). To help protect the health and safety of the public, responders, and clean-up workers during response and recovery operations from hurricanes and floods, CDC and ATSDR have developed public health guidance and other resources; many are available in both English and Spanish (Table 2).

Hurricane Harvey made landfall on the Texas coast on August 25, 2017, as a Category 4 storm. In southeast Texas, record rainfall caused extensive flooding and damage to public infrastructure and communities, and displaced thousands of persons. As of September 12, 2017, the media have reported $>80$ storm-related deaths attributed to Hurricane Harvey (medical examiner confirmation is pending for some deaths). Most of these deaths likely were caused by drowning in flood waters within the first few days after impact (e.g., drowning at home or in vehicles).

On September 7, 2017, a Category 5 hurricane, Irma, reached the Lesser Antilles, including the U.S. territories of Puerto Rico and the Virgin Islands. Hurricane Irma then continued its path across the Greater Antilles and made landfall in south Florida on September 10, 2017. Irma’s hurricane-force winds and related storm surges caused substantial damage in the Caribbean and Florida.

Many areas in Texas, Louisiana, Florida, Georgia, and the U.S. territories affected by these storms are still experiencing disruptions in essential services, including electricity, potable water, food, and communications. Numerous health care and public health systems sustained damage. Environmental health impacts from the hurricanes included effects on industries, chemical plants, and hazardous waste sites. Many displaced persons remain in shelters or other temporary housing.

As part of the overall U.S. Department of Health and Human Services response and recovery operations, CDC and ATSDR are supporting public health and medical care functions for affected communities and persons displaced by the hurricanes. As of September 12, 2017, CDC and ATSDR had sent pharmacy and federal medical station supplies to Texas, Louisiana, and Florida. CDC and ATSDR have also activated and deployed members of the U.S. Public Health Service
Commissioned Corps and other personnel to provide technical support for critical public health functions. Field operations and the CDC and ATSDR Emergency Operations Center are supporting mortality and morbidity surveillance; public health messaging and risk communication; water, sanitation, safety, and facility assessments; community rapid needs assessments; mold abatement; industrial and residential contaminant exposure prevention; and vector control.

There are potential public health and safety concerns after hurricane impact. Many injuries and illnesses from hurricanes and floods occur during the response and recovery phases. Common hazards include vehicle- and nonvehicle-related drowning, carbon monoxide poisoning (e.g., from any gasoline-powered engine, including generators and clean-up equipment), electrocution, falls, lacerations, and exposure to mold and industrial and household chemicals (1-8). In addition, exacerbation of existing chronic conditions and development of acute mental health symptoms are frequent reasons for seeking health care services following a disaster (9-11). Guidance and other resources to assist in addressing many of these hazards and risk are available (Table 2).

CDC and ATSDR also offer a disaster response clinical consultation service to assist health care providers, public health professionals, and emergency response partners. This service can be accessed by emailing CDC IMS Clinical Inquiries at eocevent168@cdc.gov.

For additional assistance, health care providers, public health professionals, and members of the public can also use CDC and ATSDR's information service, CDC-INFO. Live agents provide up-to-date science-based health information. CDC-INFO can be reached Monday through Friday from 8:00 a.m. to 8:00 p.m. Eastern Time at 1-800-CDC-INFO (1-800-232-4636) or by submitting a web-based form (https://wwwn.cdc.gov/dcs/ContactUs/Form). Services are available in English and Spanish.

\section{Conflict of Interest}

No conflicts of interest were reported.

\footnotetext{
${ }^{1}$ Emergency Operations Center, CDC.

Corresponding author: CDC Joint Information Center Administrator, eocjicad@cdc.gov.
} 
TABLE 1. English and Spanish community guidance for preparing for hurricanes and floods - CDC, 2017

English

Information about hurricanes and other tropical storms

https://www.cdc.gov/disasters/hurricanes/index.html

Preparations before a hurricane

https://www.cdc.gov/disasters/hurricanes/before.html

Family, health, and safety preparation

https://www.cdc.gov/disasters/hurricanes/supplies.html

Key facts about flood readiness

https://www.cdc.gov/disasters/floods/readiness.html

\section{En Español}

Huracanes y otras tormentas tropicales

https://www.cdc.gov/es/disasters/hurricanes/index.html

Antes de un huracán

https://www.cdc.gov/es/disasters/hurricanes/before.html

Obtenga suministros

https://www.cdc.gov/es/disasters/hurricanes/supplies.html

Datos importantes sobre los preparativos para una inundación

https://www.cdc.gov/es/disasters/floods/readiness.html

TABLE 2. English and Spanish guidance for response and recovery from hurricanes and floods, by primary target audience — CDC, 2017

\begin{tabular}{lll} 
English En Español & \\
\hline & &
\end{tabular}

\section{General audience}

Be safe after a hurricane*

https://www.cdc.gov/disasters/hurricanes/be-safe-after.html

After a hurricane

https://www.cdc.gov/disasters/hurricanes/after.html

Floods (general information)

https://www.cdc.gov/disasters/floods/index.html

After a Flood

https://www.cdc.gov/disasters/floods/after.html

Flood waters or standing waters health risks

https://www.cdc.gov/healthywater/emergency/extreme-weather/floodsstandingwater.html

Building and facilities damage: health risks

https://www.cdc.gov/healthywater/emergency/extreme-weather/buildingdamage.html

Cleaning up your home after a disaster or emergency

https://www.cdc.gov/disasters/hurricanes/cleanup-home.html

Generator and furnace safety

https://www.cdc.gov/co/pdfs/Generators.pdf

https://www.cdc.gov/co/pdfs/Furnace.pdf

Pressure washer safety

https://www.cdc.gov/disasters/pressurewashersafety.html

Carbon monoxide poisoning $\$$

https://www.cdc.gov/co/pdfs/Flyer_Danger.pdf

Carbon monoxide poisoning FAQs

https://www.cdc.gov/co/faqs.htm

Chemical hazards: asbestos in your environment: what you can do to

limit exposure

https://www.atsdr.cdc.gov/docs/limitingenvironmentalexposures_

factsheet-508.pdf

ToxFAQs for asbestos

https://www.atsdr.cdc.gov/toxfaqs/tf.asp?id=29\&tid=4

Chemical hazards: mercury

https://www.atsdr.cdc.gov/dontmesswithmercury/index.html

Chemical hazards: lead

https://www.cdc.gov/nceh/lead/tips.htm

Coping with a disaster or traumatic event

https://emergency.cdc.gov/coping/index.asp

Food safety for infants after a disaster

https://www.cdc.gov/breastfeeding/recommendations/food-safety-forinfants-after-a-disaster.html
Manténgase a salvo después de un huracán

https://www.cdc.gov/es/disasters/hurricanes/be-safe-after.html

Después de un huracán

https://www.cdc.gov/es/disasters/hurricanes/after.html

Información sobre inundaciones

https://www.cdc.gov/es/disasters/floods/index.html

Después de una inundación

https://www.cdc.gov/es/disasters/floods/after.html

Agua de la inundación después de un desastre o una emergencia: https://www.cdc.gov/es/disasters/floods/cleanupwater.html

$\boldsymbol{C}^{+}$

Limpiar tu casa después de un desastre o emergencia Limpie su casa https://www.cdc.gov/es/disasters/hurricanes/cleanup-home.html

Seguridad con los Generadores y Calentadores https://www.cdc.gov/co/pdfs/flyers_Spanish.pdf

Intoxicación por monóxido de carbono https://www.cdc.gov/co/pdfs/campaign_flyer_ES.pdf

Intoxicación con Monóxido de Carbono Preguntas Frecuente https://www.cdc.gov/co/es/faqs.htm

-

ToxFAQs Asbesto (Amianto)

https://www.atsdr.cdc.gov/es/toxfaqs/es_tfacts61.html

No te metas con mercurio

https://www.atsdr.cdc.gov/dontmesswithmercury/es/index.html

Lo que debe saber sobre el envenenamiento del plomo

https://www.cdc.gov/nceh/lead/tools/know_the_factsspanish.pdf

Cómo enfrentar un desastre o evento traumático

https://emergency.cdc.gov/es/coping/index.asp

Asegúrese de que los alimentos y el agua se puedan consumir sin correr riesgo

(Cómo alimentar a su bebé)

https://www.cdc.gov/es/disasters/hurricanes/foodwater.html

See table footnotes on page 4 
TABLE 2. (Continued) English and Spanish guidance for response and recovery from hurricanes and floods, by primary target audience CDC, 2017

\section{English}

Keep food and water safe after a disaster

https://www.cdc.gov/disasters/foodwater/facts.htm

Personal hygiene and handwashing after a disaster or emergency

https://www.cdc.gov/disasters/floods/sanitation.html

Extreme heat

https://www.cdc.gov/disasters/extremeheat/index.html

Homeowner's and renter's guide to mold cleanup after disasters

https://www.cdc.gov/mold/pdfs/homeowners_and_renters_guide.pdf

Get rid of mold

https://www.cdc.gov/disasters/hurricanes/pdf/flyer-get-rid-of-mold.pdf

Mold FAQs

https://www.cdc.gov/mold/faqs.htm

Ready Wrigley Prepares for Storm and Flood Recovery (a resource for children) https://www.cdc.gov/phpr/readywrigley/documents/17_279940_Ready_ Wrigley_mold_508.pdf

More resources for families

https://www.cdc.gov/disasters/hurricanes/more-resources.html

Public service announcements (PSAs)

https://www.cdc.gov/disasters/hurricanes/psa.html

\section{Health care professionals}

Medical care of ill disaster evacuees: additional diagnoses to consider https://www.cdc.gov/disasters/medcare.html

Medical management and patient advisement after a disaster https://www.cdc.gov/disasters/management.html

Clinical guidance for carbon monoxide (CO) poisoning after a disaster https://www.cdc.gov/disasters/co_guidance.html

Safety information for health care professionals

https://www.cdc.gov/disasters/hurricanes/hcp.html

\section{Public health professionals and response workers}

Emergency: response resources for storm, flood, and hurricane response https://www.cdc.gov/niosh/topics/emres/flood.html

Death scene investigation after natural disaster or other weather-related events: a toolkit

https://www.cdc.gov/nceh/hsb/disaster/docs/DeathScenelnvestigation508.pdf

Public health assessment and surveillance after a disaster

https://www.cdc.gov/disasters/surveillance/

Community Assessment for Public Health Emergency Response (CASPER)

https://www.cdc.gov/nceh/hsb/disaster/casper/

Emergency Responder Health Monitoring and Surveillance (ERHMS) https://www.cdc.gov/niosh/erhms/default.html

Assessment of Chemical Exposures (ACE) toolkit https://www.atsdr.cdc.gov/ntsip/ace_toolkit.html

Chemical hazards: lead information for workers https://www.cdc.gov/niosh/topics/lead/safe.html

Chemical hazards: resources for emergency responders for chemical or radioactive materials

https://www.cdc.gov/niosh/topics/emres/chemagent.html

https://www.atsdr.cdc.gov/substances/ToxEmergency.asp

Preventing carbon monoxide poisoning from small gasoline-powered engines and tools

https://www.cdc.gov/niosh/docs/96-118/

\section{En Español}

Asegúrese de que los alimentos y el agua se puedan consumir sin correr riesgo https://www.cdc.gov/es/disasters/hurricanes/foodwater.html

Higiene personal y lavado de manos después de un desastre o emergencia https://www.cdc.gov/es/disasters/floods/sanitation.html

Calor Extremo y Su Salud

https://www.cdc.gov/extremeheat/espanol/index_esp.htm

Guía del propietario y arrendatario para la limpieza de moho después de desastres

https://www.cdc.gov/mold/pdfs/IEPWG_Mold_Homeowners_and_Renters_ Spanish_508.pdf

Elimine el moho

https://www.cdc.gov/es/disasters/hurricanes/pdf/flyer-get-rid-of-mold.pdf

Preguntas más frecuentes sobre molde

https://www.cdc.gov/mold/es/faqs.htm

Más recursos para las familias

https://www.cdc.gov/es/disasters/hurricanes/more-resources.htm

Anuncios de servicio público (PSA)

https://www.cdc.gov/es/disasters/hurricanes/psa.html

-

Directrices clínicas para la intoxicación por monóxido de carbono (CO) después de un desastre https://www.cdc.gov/es/disasters/co_guidance.html

Información de seguridad para los profesionales de la salud https://www.cdc.gov/es/disasters/hurricanes/hcp.html

NIOSH advierte sobre los peligros de limpieza después de una inundación https://www.cdc.gov/spanish/NIOSH/docs/94-123_sp/

$-$

$-$

Instituto Nacional para la Seguridad y Salud Ocupacional (NIOSH) Plomo https://www.cdc.gov/spanish/niosh/topics/plomo.htm

Seguridad de productos químicos

https://www.cdc.gov/spanish/niosh/topics/quimicos.html

Prevención de envenenamiento con monóxido de carbono producido por herramientas y equipos con motores pequeños de gasoline https://www.cdc.gov/spanish/niosh/docs/96-118_sp/

See table footnotes on page 4 . 
TABLE 2. (Continued) English and Spanish guidance for response and recovery from hurricanes and floods, by primary target audience CDC, 2017

\begin{tabular}{|c|c|}
\hline English & En Español \\
\hline $\begin{array}{l}\text { Heat and outdoor workers } \\
\text { https://www.cdc.gov/disasters/extremeheat/workers.html }\end{array}$ & $\begin{array}{l}\text { Los trabajadores al aire libre y el calor } \\
\text { https://www.cdc.gov/extremeheat/espanol/workers_esp.html }\end{array}$ \\
\hline $\begin{array}{l}\text { Indoor environmental quality } \\
\text { https://www.cdc.gov/niosh/topics/indoorenv/ }\end{array}$ & - \\
\hline $\begin{array}{l}\text { Indoor environmental quality: preventing occupational respiratory disease } \\
\text { from exposures caused by dampness in office buildings, schools, and other } \\
\text { nonindustrial buildings } \\
\text { https://www.cdc.gov/niosh/docs/2013-102/ }\end{array}$ & $\begin{array}{l}\text { Prevención de enfermedades respiratorias ocupacionales por exposición } \\
\text { causadas por la humedad en edificios de oficinas, escuelas y otros edificios } \\
\text { no industriales } \\
\text { https://www.cdc.gov/spanish/niosh/docs/2013-102_sp/ }\end{array}$ \\
\hline $\begin{array}{l}\text { Indoor environmental quality: recommendations for the cleaning and } \\
\text { remediation of flood-contaminated HVAC systems: a guide for building } \\
\text { owners and managers } \\
\text { https://www.cdc.gov/niosh/topics/emres/Cleaning-Flood-HVAC.html }\end{array}$ & - \\
\hline $\begin{array}{l}\text { Safety: guidance on personal protective equipment and clothing for flood } \\
\text { cleanup workers } \\
\text { https://www.cdc.gov/niosh/topics/emres/ppe-flood.html }\end{array}$ & $\begin{array}{l}\text { Equipo de protección personal y la ropa para las personas que trabajan en la } \\
\text { limpieza después de las inundaciones } \\
\text { https://www.cdc.gov/spanish/niosh/topics/flood_sp/ppe-flood_sp.html }\end{array}$ \\
\hline $\begin{array}{l}\text { Safety: information for response and cleanup workers } \\
\text { https://www.cdc.gov/disasters/hurricanes/workers.html }\end{array}$ & $\begin{array}{l}\text { Información de seguridad para trabajadores de respuesta a emergencias y } \\
\text { de limpieza } \\
\text { https://www.cdc.gov/es/disasters/hurricanes/workers.html }\end{array}$ \\
\hline $\begin{array}{l}\text { Worker safety after a flood } \\
\text { https://www.cdc.gov/disasters/floods/workersafety.html }\end{array}$ & $\begin{array}{l}\text { Seguridad de los trabajadores después de una inundación } \\
\text { https://www.cdc.gov/es/disasters/floods/workersafety.html }\end{array}$ \\
\hline $\begin{array}{l}\text { Traumatic incident stress: symptoms and recommendations for responders } \\
\text { https://www.cdc.gov/niosh/topics/traumaticincident/ }\end{array}$ & $\begin{array}{l}\text { Estrés por sucesos traumáticos Información para el personal de emergencia } \\
\text { https://www.cdc.gov/spanish/niosh/docs/2002-107_sp/ }\end{array}$ \\
\hline $\begin{array}{l}\text { Tree removal: preventing chain saw injuries during tree removal after } \\
\text { a disaster } \\
\text { https://www.cdc.gov/disasters/chainsaws.html }\end{array}$ & $\begin{array}{l}\text { Cómo prevenir lesiones causadas por motosierras después de un desastre } \\
\text { https://www.cdc.gov/es/disasters/psa/chainsaw.html }\end{array}$ \\
\hline $\begin{array}{l}\text { Tree removal: preventing falls and electrocutions during tree trimming } \\
\text { https://www.cdc.gov/niosh/docs/92-106/ }\end{array}$ & $\begin{array}{l}\text { Retiro de árbol: prevención de caídas y electrocuciones durante la poda } \\
\text { de árboles } \\
\text { https://www.cdc.gov/spanish/niosh/docs/92-106_sp/ }\end{array}$ \\
\hline
\end{tabular}

* Information on this webpage is available in 11 different languages.

† Currently not available in Spanish.

$\S$ This fact sheet is available in six additional languages, available at https://www.cdc.gov/co/factsheets.htm.

\section{References}

1. Brandt M, Brown C, Burkhart J, et al. Mold prevention strategies and possible health effects in the aftermath of hurricanes and major floods. MMWR Recomm Rep 2006;55(No. RR-8).

2. CDC. Deaths associated with Hurricane Sandy-October-November 2012. MMWR Morb Mortal Wkly Rep 2013;62:393-7.

3. Johanning E, Auger P, Morey PR, Yang CS, Olmsted E. Review of health hazards and prevention measures for response and recovery workers and volunteers after natural disasters, flooding, and water damage: mold and dampness. Environ Health Prev Med 2014;19:93-9. https://doi. org/10.1007/s12199-013-0368-0

4. Kim S, Kulkarni PA, Rajan M, et al. Hurricane Sandy (New Jersey): mortality rates in the following month and quarter. Am J Public Health 2017;107:1304-7. https://doi.org/10.2105/AJPH.2017.303826

5. Marshall EG, Lu SE, Shi Z, Swerdel J, Borjan M, Lumia ME. Workrelated unintentional injuries associated with Hurricane Sandy in New Jersey. Disaster Med Public Health Prep 2016;10:394-404. https://doi. org/10.1017/dmp.2016.47

6. Ragan P, Schulte J, Nelson SJ, Jones KT. Mortality surveillance: 2004 to 2005 Florida hurricane-related deaths. Am J Forensic Med Pathol 2008;29:148-53. https://doi.org/10.1097/PAF.0b013e318175dd5e
7. Sengul H, Santella N, Steinberg LJ, Cruz AM. Analysis of hazardous material releases due to natural hazards in the United States. Disasters 2012;36:723-43. https://doi.org/10.1111/j.1467-7717.2012.01272.x

8. Wang A, Issa A, Bayleyegn T, et al.; Hurricane Matthew Incident Management System Team, CDC Emergency Operations Center. Notes from the field: mortality associated with Hurricane MatthewUnited States, October 2016. MMWR Morb Mortal Wkly Rep 2017;66:145-6. https://doi.org/10.15585/mmwr.mm6605a3

9. Bell SA, Abir M, Choi H, Cooke C, Iwashyna T. All-cause hospital admissions among older adults after a natural disaster. Ann Emerg Med. Epub August 5, 2017.

10. Noe RS, Schnall AH, Wolkin AF, et al. Disaster-related injuries and illnesses treated by American Red Cross disaster health services during Hurricanes Gustav and Ike. South Med J 2013;106:102-8. https://doi. org/10.1097/SMJ.0b013e31827c9e1f

11. Schwartz RM, Gillezeau CN, Liu B, Lieberman-Cribbin W, Taioli E. Longitudinal impact of Hurricane Sandy exposure on mental health symptoms. Int J Environ Res Public Health 2017;14:957. https://doi. org/10.3390/ijerph14090957 